\title{
NOUVELLE
}

\section{Effets des altérations du rythme circadien sur l'évolution du cancer du sein}

Eva Hadadi ${ }^{1,2}$, Hervé Acloque ${ }^{3,4}$
> Les rythmes biologiques permettent d'adapter le fonctionnement d'un organisme ou d'un tissu à la fluctuation de certaines conditions environnementales, comme l'alternance des saisons, des marées, ou des jours et des nuits (rythme circadien). Les périodes diurne et nocturne du rythme circadien, auxquelles correspondent les périodes d'éveil ou de veille chez les animaux selon qu'ils sont diurnes ou nocturnes, sont codées et transcrites en messages biologiques grâce à une horloge moléculaire. Chez les mammifères, cette horloge est composée d'un oscillateur central localisé dans le noyau suprachiasmatique supérieur, capable de se synchroniser grâce aux émissions lumineuses, et d'oscillateurs périphériques dans les différents tissus et organes. Ces horloges périphériques sont synchronisées sur l'horloge centrale par des voies neuronales et endocrines, mais des sti- mulations autres que la lumière, comme l'activité physique ou alimentaire, sont aussi capables de moduler ces horloges [1]. Ainsi, ces horloges interconnectées assurent un fonctionnement synchronisé des différents organes et tissus. Les horloges périphériques contrôlent l'expression circadienne de nombreux gènes (environ $10 \%$ de l'ensemble des transcrits) et permettent de moduler le fonctionnement des organes en fonction des phases d'éveil et de veille. L'horloge circadienne contrôle ainsi de nombreux processus biologiques comme le métabolisme, la prolifération, la survie et la migration cellulaires, ou encore la réponse immunitaire (Figure 1).

Des études épidémiologiques et génétiques chez l'homme ainsi que des études fonctionnelles chez la souris ont montré l'existence d'un lien entre la dérégulation de l'horloge circadienne et le développement d'un ter-
${ }^{1}$ Myeloid cell immunology lab, VIB center for inflammation research, 1050 Bruxelles, Belgique.

${ }^{2}$ Lab of cellular and molecular immunology, Vrije universiteit Brussel, 1050 Bruxelles, Belgique.

${ }^{3}$ Inserm U935, Université Paris-Saclay, 7 rue Guy-Moquet, 94800 Villejuif, France.

${ }^{4}$ Université Paris-Saclay, INRAE, AgroParisTech, GABI, 78350 Jouy-en-Josas, France.

herve.acloque@inrae.fr

rain favorable au cancer [2], notamment pour les cancers du sein [3-6]. Ainsi, une perturbation chronique du rythme circadien favorise l'apparition et la croissance des tumeurs primaires dans un modèle murin de tumorigenèse mammaire spontanée [3]. Nous avons voulu savoir si un tel dérèglement affectait également le potentiel invasif et métastatique des cellules cancéreuses. Nous voulions aussi comprendre comment les propriétés des cellules cancéreuses étaient affectées par cette perturbation, soit directement par un dysfonctionnement de leur horloge circadienne intrinsèque, soit indirectement par des modifications du micro-environnement tumoral et du système immunitaire. 


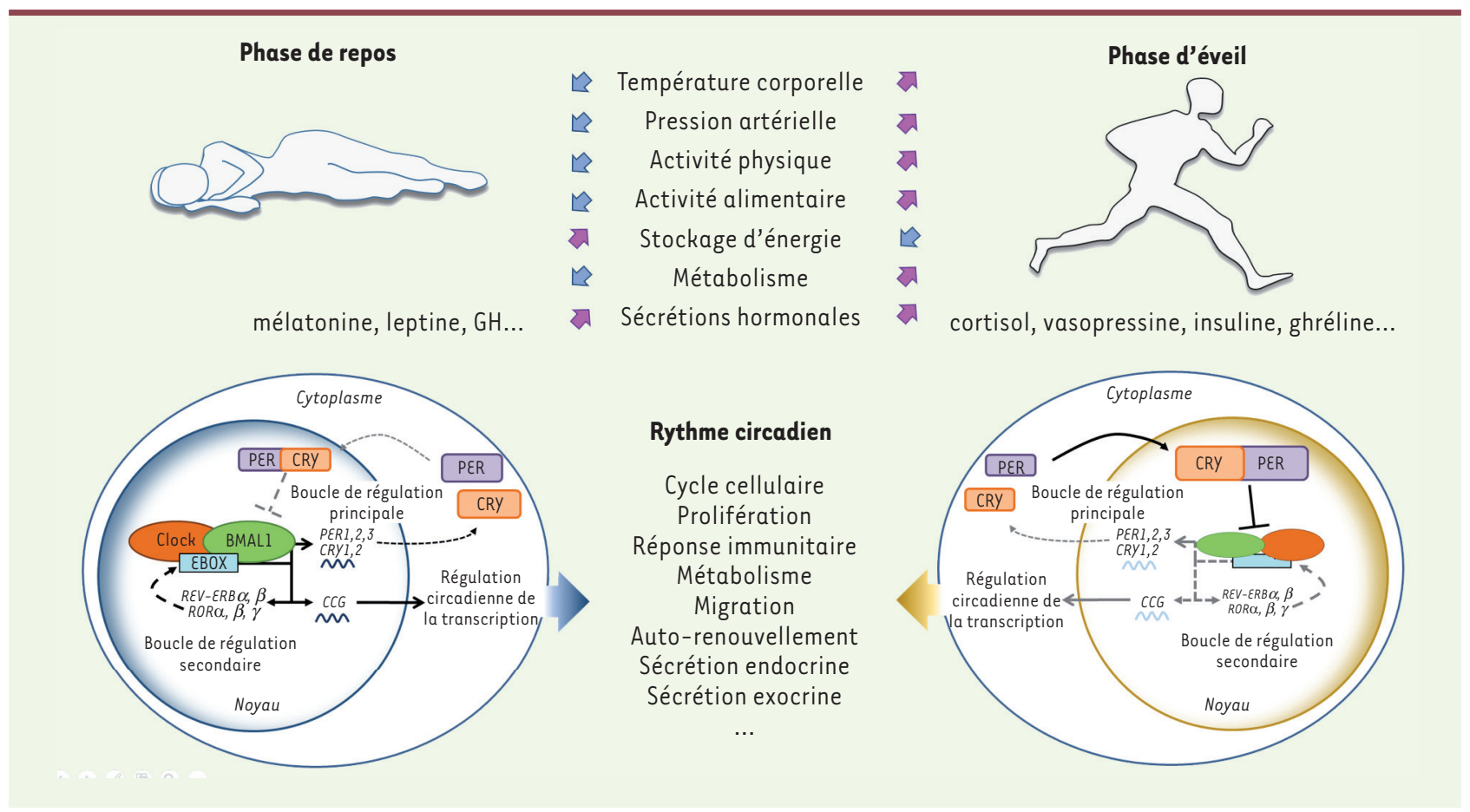

Figure 1. L'horloge circadienne contrôle le fonctionnement de l'organisme et des cellules qui le composent. En fonction des phases de repos et d'éveil, l'organisme s'adapte et favorise certaines fonctions, grâce à un dialogue hormonal et neuronal entre le système nerveux central et les organes périphériques. Les oscillations de l'horloge circadienne sont aussi interprétées à l'échelle cellulaire dans chaque organe et tissu. Durant la phase de repos, les facteurs de transcription BMALI et CLOCK s'associent, dans le noyau des cellules, en un hétérodimère capable de se fixer sur les «boîtes $\varepsilon$ » $(\varepsilon B O X)$ présentes dans les promoteurs de gènes cibles et d'induire leur transcription. On peut regrouper ces gènes cibles en deux catégories : ceux qui régulent l'horloge circadienne (gènes $P \varepsilon R, C R Y, R \varepsilon V-\varepsilon R B, R O R$ ), et ceux qui contrôlent le fonctionnement des cellules (clockcontrolled genes, CCG). L'activation des gènes $P \varepsilon R$ et $C R Y$ entraîne la mise en place d'une boucle de régulation négative et enclenche la transition vers la phase d'éveil. Durant la phase d'éveil, les protéines PER et CRY répriment l'activité transcriptionnelle induite par le dimère CLOCK-BMALI. Cela active à nouveau la transcription de BMALI et CLOCK pour enclencher la transition vers la phase de repos. Cela entraîne aussi une diminution de l'expression des gènes CCG. Ces oscillations transcriptionnelles modulent donc de nombreuses fonctions cellulaires comme la prolifération et le métabolisme cellulaires, les sécrétions exocrines et endocrines, ou les migrations et infiltrations de cellules immunitaires.

Nous avons donc exposé des souris développant spontanément des tumeurs mammaires à la puberté (modèle MMTVPyMT) [7] à un jet lag chronique durant 10 semaines, consistant, sur la base d'un rythme jour/nuit de 12 heures/12 heures, à réduire de 8 heures la durée de la nuit toutes les deux nuits, afin de reproduire les perturbations circadiennes induites par le travail de nuit en rotation [8]. Nous avons ainsi montré que l'exposition à un stress circadien chronique concomitant à la progression tumorale augmente significativement la dissémination des cellules cancéreuses mammaires dans l'organisme [9]. Nous avons ensuite cherché à comprendre comment cette perturbation circadienne chronique augmentait le potentiel métastatique des cellules cancéreuses. Pour cela, nous avons orienté nos recherches sur trois mécanismes majeurs de la progression tumorale: l'émergence des cellules souches cancéreuses, l'activation de la transition épithélio-mésenchymateuse, qui favorise la dissémination des cellules cancéreuses, et le développement d'un microenvironnement pro-tumoral. Ces trois mécanismes pourraient être liés et dépendre du rythme circadien [10].

Après le jet lag imposé aux souris, nous avons observé une augmentation du nombre de cellules cancéreuses porteuses d'une signature de cellules souches mammaires. Cette augmentation était associée à une capacité accrue à former des mammosphères in vitro et à provoquer des tumeurs à la suite d'une greffe in vivo. Nos observations montrent donc qu'un stress circadien chronique favorise l'émergence des cellules souches cancéreuses dans les tumeurs primaires mammaires. Comme nous avions également observé une dissémination accrue des cellules cancéreuses, et que l'émergence des cellules souches cancéreuses est favorisée par l'activation de la transition épithéliomésenchymateuse, nous avons également recherché des marqueurs de cette transition dans les tumeurs primaires. Après le jet lag, nous avons détecté une expression accrue de facteurs induisant la transition épithélio-mésenchyma- 
Vieillissement

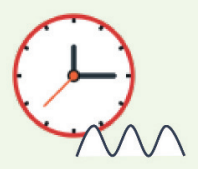

Mutations germinales/somatiques

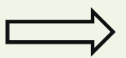

Travail de nuit/Jet lag

Troubles comportementaux

(sommeil, alimentation)

Progression tumorale facilitée par :

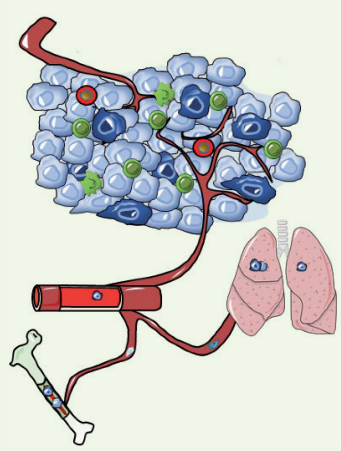

$$
\begin{gathered}
\text { Immuno-suppression } \\
\text { Cellules souches } \\
\text { cancéreuses } \\
\text { Inflammation } \\
\text { Invasion/Dissémination }
\end{gathered}
$$
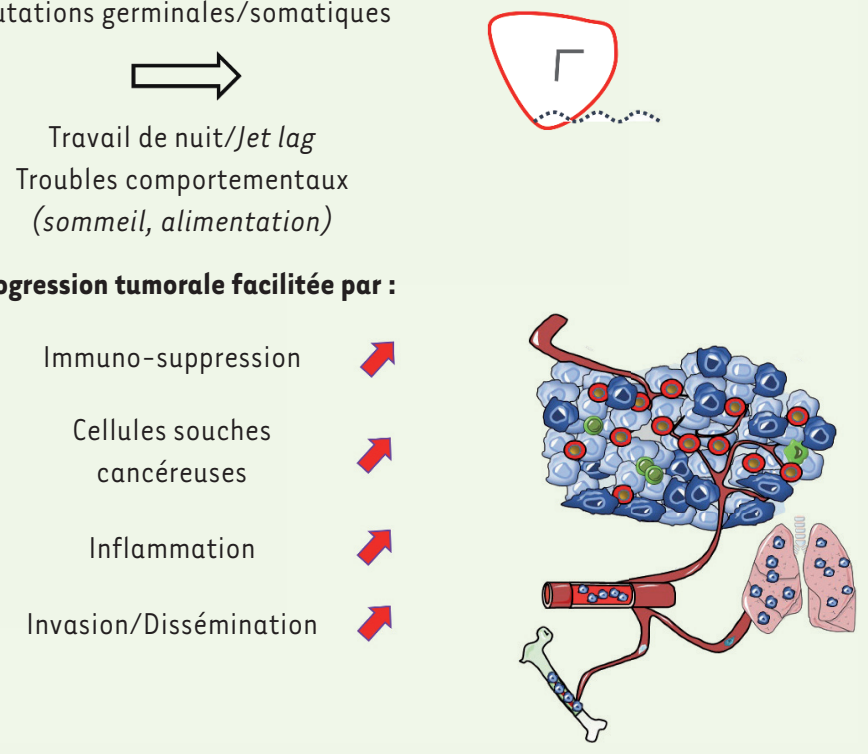

Figure 2. Effets de modifications du rythme circadien sur la progression et la malignité du cancer du sein. Une altération de l'horloge circadienne, induite par des facteurs environnementaux, sociétaux ou génétiques, a une répercussion sur la progression des tumeurs mammaires. Notre étude sur des souris développant spontanément des tumeurs mammaires a montré qu'un jet lag chronique modifie le microenvironnement tumoral, en favorisant l'inflammation locale et le recrutement de cellules immunitaires pro-tumorales (en rouge), et en diminuant le nombre de cellules immunitaires anti-tumorales (en vert). Ce jet lag chronique affecte aussi les propriétés des cellules cancéreuses en augmentant la proportion de cellules souches cancéreuses (en bleu foncé) et en facilitant leur dissémination dans l'organisme, aussi bien dans le sang, la moelle osseuse et les os, que dans les poumons.

teuse, comme les facteurs de transcription Zeb2 et FoxC2, associée à une plus grande agressivité tumorale, confirmée par l'analyse immunohistologique avec les marqueurs CKl4, vimentine et fibronectine. Ces résultats montrent qu'un dérèglement chronique de l'horloge circadienne affecte les propriétés intrinsèques des cellules cancéreuses, et modifie leurs potentiels de prolifération, d'invasion et de dissémination.

Mais notre étude montre aussi que le dérèglement de l'horloge circadienne affecte le microenvironnement tumoral et favorise un contexte immunitaire protumoral. Nous avons ainsi observé que certaines populations de cellules immunitaires infiltrées étaient modifiées, notamment les lymphocytes T CD8 ${ }^{+}$ cytotoxiques, dont la proportion diminue. Les populations de macrophages associés aux tumeurs sont également modifiées, notamment les populations pro-tumorales, immunosuppressives, qui sont augmentées (Figure 2).

Afin de déterminer l'origine moléculaire de ces changements, nous avons mesuré les cytokines et chimiokines impliquées dans le recrutement et l'activation des populations de cellules immunitaires. Nous avons notamment constaté que la signalisation CXCL5 ( $C-X-C$ motif chemokine 5) / CXCR2 ( $C-X-C$ motif chemokine receptor 2 ) était augmentée, ce qui pourrait expliquer le recrutement des macrophages immunosuppresseurs dans le microenvironnement tumoral. Pour valider expérimentalement cette observation, nous avons injecté un inhibiteur du récepteur CXCR2 à des souris soumises au jet lag. Nous avons alors constaté des changements significatifs du microenvironnement tumoral, associés à une diminution du poten- tiel métastatique des cellules cancéreuses. Le nombre des macrophages protumoraux diminue, alors que celui des lymphocytes $\mathrm{T}$ cytotoxiques augmente, contribuant ainsi à diminuer le nombre de cellules cancéreuses disséminées.

Notre étude a permis de montrer les effets délétères d'un dérèglement global de l'horloge circadienne, à l'échelle de l'organisme, sur la progression tumorale des cancers mammaires. Un tel dérèglement affecte notamment les propriétés intrinsèques des cellules cancéreuses, les rendant plus agressives, mais il modifie également le microenvironnement tumoral et les cellules immunitaires qui le composent. Cela conduit à la mise en place d'une immunosuppression locale protégeant les cellules cancéreuses de leur destruction par le système immunitaire (Figure 2) [9]. Nos résultats soulignent l'importance du 
rythme circadien dans le développement et la progression des cancers du sein. Il paraît donc judicieux de renforcer les mesures de prévention du cancer du sein et son dépistage chez les femmes qui seraient particulièrement exposées à un dérèglement de l'horloge circadienne, par leurs activités professionnelles, les conséquences d'autres maladies (notamment celles provoquant des troubles du sommeil, de l'humeur, ou du comportement alimentaire), l'exposition à un environnement particulier (comme les nuisances sonores), ou encore par la présence de mutations génétiques affectant le fonctionnement de cette horloge. Favoriser le retour à un rythme circadien physiologique permettrait également d'obtenir une meilleure efficacité de la chimiothérapie et de l'immunothérapie contre ces tumeurs. Nous envisageons donc maintenant d'évaluer le rôle bénéfique d'une chrono-modulation pharmacologique débutée préalablement aux traitements conventionnels du cancer du sein, afin de favoriser le rétablissement rapide d'une horloge circadienne fonctionnelle.

\section{Effects of circadian rhythm disorders} on breast cancer progression

\section{LIENS D'INTÉRÊT}

Les auteurs déclarent n'avoir aucun lien d'intérêt concernant les données publiées dans cet article.

\section{RÉFÉRENCES}

1. Panda S. Circadian physiology of metabolism. Science 2016 ; 354 : 1008-15

2. Sulli G, Lam MTy, Panda S. Interplay between circadian clock and cancer: new frontiers for cancer treatment. Trends Cancer $2019 ; 8: 475-94$.

3. Van Dycke KCG, Rodenburg W, van Oostrom CTM, et al. Chronically alternating light cycles increase breast cancer risk in mice. Curr Biol $2015 ; 25$ : 1932-7.
4. Cordina-Duverger $\varepsilon$, Menegaux F, Popa A, et al. Night shift work and breast cancer: a pooled analysis of population-based case-control studies with complete work history. Eur J Epidemiol 2018 ; 33 : 369-79.

5. Mocellin S, Tropea S, Benna C, Rossi CR. Circadian pathway genetic variation and cancer risk: evidence from genome-wide association studies. BMC Med 2018 ; 16 : doi: 10.1186/s12916-018-1010-1. 20.

6. McQueen CM, Schmitt દE, Sarkar TR, et al. PER2 regulation of mammary gland development. Development 2018 ; 145. doi : 10.1242/dev157966.

7. Guy CT, Cardiff RD, Muller WJ. Induction of mammary tumors by expression of polyomavirus middle $T$ oncogene: a transgenic mouse model for metastatic disease. Mol Cell Biol $1992 ; 12$ : 954-61.

8. Filipski $\varepsilon$, Delaunay F, King VM, et al. Effects of chronic jet lag on tumor progression in mice. Cancer Res $2004 ; 64: 7879-85$.

9. Hadadi $\varepsilon$, Taylor W, Li XM, et al. Chronic circadian disruption modulates breast cancer stemness and immune microenvironment to drive metastasis in mice. Nat Commun 2020 ; 11 : doi: 10.1038/s41467020-16890-6.

10. Hadadi $\varepsilon$, Acloque $H$. Role of circadian rhythm disorders in EMT and tumor-immune interactions in endocrine-related cancers. Endocr Relat Cancer $2021 ; 28$ : R67-80. 